\title{
THE ORBITAL ECCENTRICITIES OF BINARY MILLISECOND PULSARS IN GLOBULAR CLUSTERS
}

\author{
FREDERIC A. RASIO \\ Dept of Physics, MIT \\ AND \\ DOUGLAS C. HEGGIE \\ Dept of Mathematics and Statistics, Univ. of Edinburgh
}

\begin{abstract}
Low-mass binary millisecond pulsars are born with very small orbital eccentricities, typically of order $e_{i} \sim 10^{-6}-10^{-3}$. In globular clusters, however, higher eccentricities $e_{f} \gg e_{i}$ can be induced by dynamical interactions with passing stars. Using both analytical perturbation calculations and numerical integrations, we have shown (Heggie \& Rasio 1996) that the cross section for this process is much larger than previously estimated. This is because, even for initially circular binaries, the induced eccentricity $e_{f}$ for an encounter with pericentre separation $r_{\mathrm{p}}$ beyond a few times the binary semi-major axis $a$ declines only as a power-law, $e_{f} \propto\left(r_{\mathrm{p}} / a\right)^{-5 / 2}$, and not as an exponential. We find that all currently known low-mass binary millisecond pulsars in globular clusters must have been affected by interactions, with their current eccentricities being at least an order of magnitude larger than at birth (Rasio \& Heggie 1995).
\end{abstract}

\section{References}

Heggie D.C., Rasio F.A., 1996, MNRAS, submitted

Rasio F.A., Heggie D.C., 1995, ApJ, 445, L133 